\title{
ESTUDO ELETROMIOGRÁFICO DOS MÚSCULOS FACIAIS DE RESPIRADORES NASAIS, RESPIRADORES ORAIS VICIOSOS E OBSTRUTIVOS
}

\section{Electromyographic study on facial muscles of nasal breathers, obstructive and vicious oral breathers}

\author{
Luane de Moraes Boton (1), Ana Maria Toniolo da Silva (2), Geovana de Paula Bolzan (3), \\ Eliane Castilhos Rodrigues Corrêa ${ }^{(4)}$, Angela Ruviaro Busanello ${ }^{(5)}$
}

\begin{abstract}
RESUMO
Objetivo: verificar a atividade elétrica dos músculos orbiculares orais, masseteres e temporais no repouso, nas isometrias, labial e mastigatória, em crianças respiradoras nasais e respiradoras orais viciosas e obstrutivas, comparando-as. Métodos: foram estudadas 59 crianças, 15 respiradoras nasais $(\mathrm{RN})$; 23 respiradoras orais viciosas $(\mathrm{ROV})$ e 21 respiradoras orais obstrutivas $(\mathrm{ROO})$. Todas foram submetidas à avaliação otorrinolaringológica, à fonoaudiológica e ao exame eletromiográfico durante repouso, isometrias mastigatória e labial. Foi realizada análise de variância de Kruskal-Wallis para comparação entre os grupos e dos grupos dois a dois e o teste de Wilcoxon para comparação entre os músculos $(p<0,05)$. Resultados: quando se comparou os três grupos, não houve diferença significativa nos músculos estudados, exceto no músculo masseter direito durante repouso, quando se comparou os RN e ROV; para os músculos orbicular inferior no repouso e temporal esquerdo na isometria mastigatória, quando se comparou os RN e ROO. Na comparação dos ROV e ROO nenhum músculo mostrou diferença significativa. $\mathrm{O}$ músculo orbicular inferior se mostrou mais ativo que orbicular superior no repouso e na isometria labial, principalmente, nos ROV e ROO. A comparação dos músculos dos lados opostos da face na isometria mastigatória mostrou assimetria. O músculo temporal apresentou-se mais ativo que os masseteres no repouso e na isometria mastigatória. Conclusão: o modo respiratório não modificou o comportamento dos músculos avaliados quando realizadas comparações entre os grupos e as diferentes etiologias da respiração oral não alteraram a atividade elétrica dos músculos avaliados quando se comparou os ROV e ROO.
\end{abstract}

DESCRITORES: Eletromiografia; Respiração Bucal; Músculos Faciais

(1) Fonoaudióloga; Mestranda em Distúrbios da Comunicação Humana pela Universidade Federal de Santa MariaUFSM, RS.

(2) Fonoaudióloga; Professor Associado do Departamento de Fonoaudiologia da Universidade Federal de Santa Maria, RS; Doutora em Ciências dos Distúrbios da Comunicação Humana pela Universidade Federal de São Paulo - UNIFESP, SP.

(3) Fonoaudióloga; Mestranda em Distúrbios da Comunicação Humana pela Universidade Federal de Santa Maria UFSM, RS.

(4) Fisioterapeuta; Doutora em Biologia Buco-Dental (área de Anatomia) pela Universidade Esatdual de Campinas - UNICAMP, Piracicaba/SP.

(5) Fonoaudióloga; Mestre em Distúrbios da Comunicação Humana pela Universidade Federal de Santa Maria UFSM, RS.

Conflito de interesses: inexistente

\section{INTRODUÇÃO}

A respiração nasal favorece o crescimento e desenvolvimento facial harmônico; porém, quando essa é substituída pela respiração oral, deve ser considerada uma condição patológica por acarretar diversas alterações morfofuncionais no sistema estomatognático. Dentre as principais alterações estão deglutição alterada, mastigação ineficiente, fala distorcida, além de postura inadequada dos órgãos fonoarticulatórios e hipotonia muscular ${ }^{1-3}$.

É fundamental o conhecimento da etiologia da respiração oral, a qual pode ser dividida em duas categorias: obstrutiva, decorrente de algum impedimento mecânico a passagem de ar, como desvio 
de septo, hiperplasia das tonsilas faríngeas ou palatinas (adenóide e/ou amígdala) ${ }^{4}$; e não obstrutiva ou viciosa, quando ocorre por flacidez dos órgãos fonoarticulatórios ou por simples hábito ${ }^{5,6}$.

Considerando os fatores desencadeantes da respiração oral e vários estudos ${ }^{3,5,6}$ que destacam a importância do diagnóstico da mesma, é que surgiu o interesse por esta pesquisa. Acredita-se na hipótese de que crianças com o modo respiratório oral por obstrução nasal apresentam probabilidade de desenvolverem alterações musculares mais severas do que as crianças com respiração oral viciosa. A confirmação dessa hipótese constituiria um dado importante para a conduta terapêutica.

A avaliação da atividade eletromiográfica dos músculos orbiculares orais, dos masseteres e dos temporais, fornece informação quantitativa sobre as repercussões do modo respiratório e das etiologias da respiração oral sobre esses músculos.

Sendo assim, este estudo teve como objetivo verificar a atividade elétrica dos músculos orbiculares orais, dos masseteres e dos temporais no repouso, nas isometrias labial e mastigatória em crianças respiradoras nasais e respiradoras orais viciosas e obstrutivas, comparando-as.

\section{MÉTODOS}

Esta pesquisa apresenta caráter quantitativo, transversal e contemporâneo. Foi realizada de acordo com as normas e diretrizes do Conselho Nacional de Saúde, resolução 196/1996 (Brasil Resolução MS/CNS/CNEP n. ${ }^{\circ}$ 196/96 de 10 de outubro de 1996). Participaram do estudo crianças com idades entre 7 anos e 11 anos e 11 meses, cujos pais estiveram de acordo com as explicações prestadas pela pesquisadora sobre os aspectos do estudo e haviam assinado o Termo de Consentimento Livre e Esclarecido (TCLE).

Foram excluídas crianças que (1) apresentaram sinais evidentes de comprometimento neurológico e sindrômico; (2) realizaram tratamento ortodôntico, terapia fonoaudiológica, cirurgia facial; ou ainda, (3) apresentaram malformações craniofaciais.

Participaram desta pesquisa 59 crianças de ambos os gêneros, que se enquadraram nos critérios pré-estabelecidos e cujos pais aderiram ao TCLE. Para dividir os três grupos de estudo, todas as crianças passaram por avaliação otorrinolaringológica, realizada por médico otorrinolaringologista, através de exame de nasofibroscopia, realizado com nasofibroscópio flexível $3.2 \mathrm{~mm}$, marca Machida, microcamera marca Asap e gravado em DVD. Essa avaliação teve como finalidade confirmar o modo respiratório e, nas crianças com respiração oral, verificar se a causa era obstrução nasal ou hábito (vício).

Para divisão dos grupos, foram consideradas respiradoras nasais as crianças que apresentaram respiração predominantemente nasal; respiradoras orais viciosas as crianças que apresentaram respiração predominantemente oral, sem obstrução nas vias aéreas superiores; e respiradoras orais obstrutivas as crianças que apresentaram respiração predominantemente oral com presença de hipertrofia de adenóide e/ou de amígdalas grau três ou quatro. Não ocorreram, outras patologias obstrutivas de vias aéreas superiores.

Assim, de acordo com as alterações fonoaudiológicas verificadas e com a etiologia da respiração oral, as crianças foram separadas nos seguintes grupos: grupo de respiradores nasais (RN), composto por 15 crianças, 13 do sexo feminino e duas do sexo masculino; grupo de respiradores orais viciosos (ROV), composto por 23 crianças, 10 do sexo feminino e 13 do sexo masculino; e grupo de respiradores orais obstrutivos (ROO), composto por 21 crianças, 11 do sexo feminino e 10 do sexo masculino.

Após seleção dos grupos, foi realizado o exame eletromiográfico nas situações de repouso, isometria mastigatória e isometria labial.

As crianças foram previamente treinadas durante as coletas e permaneceram sentadas confortavelmente, com a cabeça orientada de acordo com o Plano de Frankfurt. Em cada uma das situações avaliadas, foram realizadas três coletas, com o propósito de excluir possíveis induções de resultados, bem como, para assegurar a fidedignidade do exame ${ }^{7}$.

O teste de repouso foi realizado com a criança sentada, em posição relaxada de lábios e mandíbula $^{8}$, apenas com os lábios em vedamento, sem contato entre as arcadas dentárias, mantendo a postura habitual de língua, devendo permanecer assim por 10 segundos.

Durante o teste de isometria mastigatória, a criança foi orientada a morder com força máxima bilateral e simultaneamente, permanecendo assim por 5 segundos, sendo que, entre as arcadas foi colocada uma folha de parafilm (Parafilm M, Laboratory Film) medindo três $\mathrm{cm}$ de comprimento, um $\mathrm{cm}$ de largura e dobrada em cinco partes iguais ${ }^{9}$.

No teste de isometria labial, a criança deveria manter o máximo de apertamento labial, também por 5 segundos. Foi considerada adequada a compressão recíproca dos orbiculares ${ }^{10}$. Para a correta realização da prova, a criança foi submetida a treino prévio sob a observação do examinador. A escolha do melhor sinal considerou a configuração do mesmo, ou seja, o que apresentou menor sinal 
de ruído, bem como o histograma mais harmônico e coerente com sinal selecionado ${ }^{8}$.

Para o restabelecimento muscular e a não ocorrência de fadiga, as coletas foram realizadas com intervalo de dois minutos ${ }^{11}$.

As crianças tiveram a pele do rosto higienizada com álcool etílico $70 \%$, onde foram colocados eletrodos duplos de superfície de $\mathrm{Ag} / \mathrm{AgCl}$, da marca Hal Ind. e Com. Nos músculos orbiculares superior e inferior, os eletrodos foram colados nos ventres, sendo necessário, em algumas crianças, o recorte dos rebordos adesivos dos eletrodos para a adequada aderência, conforme recomendações ${ }^{11}$. Já nos músculos masseter e temporal, os eletrodos foram fixados na região de maior volume e maior massa muscular, possibilitando a captação da resposta do maior número de unidades motoras ${ }^{12}$.

Além disso, foi realizado teste de função muscular, solicitando à criança que realizasse contração voluntária máxima para a localização do músculo masseter e porção anterior do músculo temporal ${ }^{13}$. Para evitar qualquer interferência/ruído ao sinal eletromiográfico, utilizou-se um eletrodo de referência (terra) na testa do paciente (glabela). Foram desligados todos os aparelhos eletrônicos e fontes de luz. Além disso, foi realizado revestimento do piso e da mesa no local de realização das coletas com material emborrachado ${ }^{7}$.

O equipamento utilizado para realização do exame eletromiográfico foi o eletromiógrafo modelo EMG 1200 (Lynx Tecnologia Itda.), composto de oito canais de entrada, placa conversora A/D de 16 bits e faixa de entrada de $+/-2 \mathrm{~V}$. Utilizou-se 0 filtro do tipo Butterworth, freqüência de corte passaalta de $10 \mathrm{~Hz}$ e passa-baixa de $1000 \mathrm{~Hz}$, freqüência de amostragem de $2 \mathrm{KHz}, 2048$ amostras/canal e tempo de amostragem de 1024s. Os sinais eletromiográficos foram coletados por meio do Software Biolnspector 1.8 (Lynx), captados através de préamplificadores ativos com entrada diferencial (PA 1020) da Lynx Eletronics Ltda. O ganho foi de 20 vezes, a impedância de entrada de $10 \mathrm{G} \Omega$ e taxa de rejeição de modo comum $>100 \mathrm{~dB}$.

Os sinais eletromiográficos foram quantificados em RMS, expressos em $\mu \mathrm{V}$ (microvoltz) e salvos em computador portátil com bateria própria. $\mathrm{A}$ análise dos dados foi realizada pelo software AqDAnalysis 7.0 (Lynx). Optou-se pela análise do sinal em RMS sem normalização; pois, embora recomendada por permitir a reprodutibilidade dos registros, o uso da mesma pode vir a suprimir distinções entre os sinais coletados ${ }^{11}$. Além disso, considerando que a colocação dos eletrodos nos músculos estudados fica limitada a uma área restrita, a justificativa para normalização pela possibilidade de se recolocar eletrodos em áreas distintas, no mesmo músculo, em diferentes avaliações, não se aplicaria a músculos com superfície de tamanho reduzido.

Esta pesquisa foi aprovada pelo Comitê de Ética em Pesquisa da instituição no qual foi desenvolvida, sob o número 0220.0.243.000-08.

Para estudar as diferenças entre os três grupos propostos, utilizou-se a análise de variância de Kruskal-Wallis, a qual também foi empregada para realizar a comparação dos grupos de estudo dois a dois. Somando-se a isso, foi realizada uma análise intra-grupos, comparando os músculos entre si, dentro de cada função estudada. Para tal análise, foi utilizado o teste de Wilcoxon. Foi considerada significância de $5 \%(p<0,05)$ em todas as análises.

\section{RESULTADOS}

Na Tabela 1, estão expostos as médias e os desvios padrão da atividade elétrica (RMS) dos grupos estudados (Respiradores nasais- RN, Respiradores orais viciosos- ROV e Respiradores orais obstrutivos- ROO) e a comparação realizada nos três grupos nas situações de repouso, isometria mastigatória e isometria labial.

$\mathrm{Na}$ Tabela 2, encontram-se os resultados da comparação da atividade elétrica (RMS) realizadas entre os grupos dois a dois (RN $x$ ROV/ RN $x$ $\mathrm{ROO} / \mathrm{ROV} \times \mathrm{ROO}$ ) para cada uma das provas executadas.

$\mathrm{Na}$ tabela 3 , estão as comparações $(p<0,05)$ pertinentes da atividade elétrica (RMS), dos músculos orbicular superior e inferior, dos masseteres e dos temporais nos lados direito e esquerdo e entre masseteres direito e esquerdo e temporais direito e esquerdo durante o repouso, a isometria mastigatória e a isometria labial em cada um dos grupos (RN, ROV e ROO).

\section{DISCUSSÃO}

Ao realizar a comparação da atividade elétrica entre os RN, ROV e ROO, durante o repouso, com vedamento labial, e as isometrias mastigatória e labial, não foi observada diferença estatisticamente significante entre os músculos avaliados (Tabela 1). Verificou-se com estes resultados que a atividade elétrica foi semelhante nos três grupos estudados, mostrando que o modo respiratório e a etiologia da respiração oral, nesta pesquisa, não influenciaram o comportamento desses músculos. Resultado que vai de encontro com a literatura, uma vez que os respiradores orais, quando comparados com respiradores nasais, tendem a apresentar atividade elétrica inferior durante isometria ${ }^{8}$ e superior na situação de repouso ${ }^{10,13,14}$. 
Tabela 1 - Distribuição de médias e desvios padrão da atividade elétrica (RMS) dos músculos analisados durante o repouso, isometria mastigatória e isometria labial nos respiradores nasais (RN), respiradores orais viciosos (ROV) e respiradores orais obstrutivos (ROO) e comparação da atividade elétrica (RMS) entre os três grupos estudados

\begin{tabular}{|c|c|c|c|c|c|c|c|c|}
\hline \multirow{2}{*}{ Funções } & & \multicolumn{2}{|c|}{ RN } & \multicolumn{2}{|c|}{ ROV } & \multicolumn{2}{|c|}{ ROO } & \multirow{2}{*}{$\frac{R N \times R O V \times R O O}{p}$} \\
\hline & & Média & (DP) & Média & (DP) & Média & (DP) & \\
\hline \multirow{6}{*}{ Repouso } & OS & 4,40 & $(1,96)$ & 5,11 & $(3,04)$ & 5,51 & $(4,82)$ & 0,9220 \\
\hline & OI & 5,29 & $(2,74)$ & 8,28 & $(4,97)$ & 10,37 & $(8,38)$ & 0,0621 \\
\hline & MD & 3,55 & $(0,70)$ & 3,27 & $(1,71)$ & 3,47 & $(1,02)$ & 0,0511 \\
\hline & $\mathrm{ME}$ & 3,75 & $(1,34)$ & 3,66 & $(1,64)$ & 3,80 & $(1,40)$ & 0,7440 \\
\hline & TD & 3,96 & $(0,90)$ & 4,08 & $(0,99)$ & 3,96 & $(1,19)$ & 0,6607 \\
\hline & TE & 4,50 & $(0,62)$ & 4,23 & $(1,41)$ & 4,11 & $(0,98)$ & 0,1652 \\
\hline \multirow{4}{*}{$\begin{array}{c}\text { Isometria } \\
\text { Mastigatória }\end{array}$} & MD & 205,49 & $(140,56)$ & 244,18 & $(126,35)$ & 250,11 & $(127,79)$ & 0,3025 \\
\hline & $\mathrm{ME}$ & 141,60 & $(85,26)$ & 196,25 & $(103,09)$ & 189,80 & $(96,65)$ & 0,1300 \\
\hline & TD & 219,03 & $(91,45)$ & 236,38 & $(85,79)$ & 248,98 & $(100,07)$ & 0,5938 \\
\hline & TE & 198,80 & $(60,96)$ & 254,60 & $(105,73)$ & 261,52 & $(89,71)$ & 0,0851 \\
\hline \multirow{2}{*}{$\begin{array}{l}\text { Isometria } \\
\text { Labial }\end{array}$} & OS & 175,43 & $(57,18)$ & 178,15 & $(50,43)$ & 171,83 & $(40,80)$ & 0,7570 \\
\hline & OI & 325,95 & $(92,99)$ & 314,41 & $(95,06)$ & 284,84 & $(72,47)$ & 0,5886 \\
\hline
\end{tabular}

* Significância estatística pelo teste Kruskal-Wallis $(p<0,05)$.

Legenda: OS- orbicular superior/ OI- orbicular inferior/ MD- masseter direito/ ME- masseter esquerdo/ TD- temporal direito/ TE- temporal esquerdo/ DP- desvio padrão

Tabela 2 - Comparações da atividade elétrica (RMS) realizada entre os grupos dois a dois (RN x ROV/ RN x ROO/ ROV x ROO) no repouso, na isometria mastigatória e na isometria labial

\begin{tabular}{ccccc}
\hline \multirow{2}{*}{ Funções } & Músculos & RN x ROV & RN x ROO & ROV x ROO \\
\cline { 2 - 4 } & & $\mathbf{P}$ & $\mathbf{p}$ & $\mathbf{p}$ \\
\hline \multirow{3}{*}{ Repouso } & OS & 0,6868 & 0,8348 & 0,8417 \\
& OI & 0,0579 & $0,0280^{*}$ & 0,5648 \\
& MD & $0,0304^{*}$ & 0,6189 & 0,0555 \\
& ME & 0,6012 & 0,9361 & 0,4592 \\
& TD & 0,6012 & 0,7362 & 0,3782 \\
Isometria & TE & 0,1169 & 0,0698 & 0,8972 \\
\hline Mastigatória & MD & 0,2044 & 0,1443 & 0,8417 \\
& ME & 0,0620 & 0,0860 & 0,9719 \\
& TD & 0,4828 & 0,2970 & 0,7870 \\
Isometria Labial & TE & 0,1169 & $0,0257^{*}$ & 0,5491 \\
\hline
\end{tabular}

*Significância estatística pelo teste Kruskal-Wallis $(p<0,05)$.

Legenda: OS- orbicular superior/ Ol- orbicular inferior/ MD- masseter direito/ ME- masseter esquerdo/ TD- temporal direito/ TE- temporal esquerdo 
Tabela 3 - Comparações pertinentes da atividade elétrica entre os músculos: lados opostos (OS x OI/ MD x ME/ TD x TE) e mesmos lados (MD x TD/ ME x TE) nos grupos analisados

\begin{tabular}{|c|c|c|c|c|}
\hline \multirow{2}{*}{ Funções } & \multirow{2}{*}{ Relações } & RN & ROV & ROO \\
\hline & & $\mathbf{P}$ & $p$ & $\mathbf{P}$ \\
\hline \multirow{5}{*}{ Repouso } & $\mathrm{OS} \times \mathrm{OI}$ & 0,2330 & $0,0009^{* *}$ & $0,0001^{* *}$ \\
\hline & $\mathrm{MD} \times \mathrm{ME}$ & 0,9096 & 0,1209 & 0,5663 \\
\hline & TD x TE & $0,0468^{*}$ & 0,7380 & 0,6894 \\
\hline & MD x TD & 0,0609 & $0,0285^{\star}$ & 0,1138 \\
\hline & ME $\times$ TE & 0,0884 & $0,0359^{\star}$ & 0,2443 \\
\hline \multirow{4}{*}{$\begin{array}{l}\text { Isometria } \\
\text { mastigatória }\end{array}$} & $\mathrm{MD} \times \mathrm{ME}$ & $0,0106^{*}$ & $0,0051^{*}$ & $0,0006^{\star *}$ \\
\hline & TD x TE & 0,3066 & 0,1443 & 0,3570 \\
\hline & MD x TD & 0,1118 & 0,9515 & 0,8213 \\
\hline & ME $\times$ TE & $0,0090^{*}$ & $0,0062^{*}$ & $0,0005^{\star *}$ \\
\hline Isometria labial & $\mathrm{OS} \times \mathrm{OI}$ & $0,0007^{* *}$ & $0,0001^{* *}$ & $0,0001^{* *}$ \\
\hline
\end{tabular}

* Significância estatística pelo teste Wilcoxon $\left({ }^{*} \mathrm{p}<0,05\right.$-Diferença significativa/ ${ }^{* *} \mathrm{p}<0,001$ - diferença altamente significativa)

Legenda: RN- respiradores nasais/ ROV- respiradores orais viciosos/ ROO- respiradores orais obstrutivos/ OS- orbicular superior/ OI- orbicular inferior/ MD- masseter direito/ ME- masseter esquerdo/ TD- temporal direito/ TE- temporal esquerdo.

Entretanto, ao se observar os ROV e os ROO, durante o repouso, percebeu-se que os músculos orbiculares, principalmente o orbicular inferior dos ROO, apresentaram-se eletricamente mais ativos que os RN, sugerindo maior atividade deste músculo nos respiradores orais. Estudos semeIhantes também verificaram que respiradores orais apresentam a musculatura periorbicular mais ativa que a dos respiradores nasais ${ }^{10,13,14}$, na tentativa de compensar o comprometimento desta musculatura que, em geral, se apresenta hipotônica e alterada quanto ao comprimento e ao posicionamento nos respiradores orais ${ }^{3,15}$.

$\mathrm{Na}$ comparação da atividade elétrica dos músculos avaliados entre os grupos RN e ROV, nas três situações avaliadas, verificou-se que os resultados se mostraram semelhantes entre eles, com exceção do músculo masseter direito na prova de repouso. Nesse caso, foi possível observar que o músculo masseter direito dos RN apresentou atividade elétrica superior que o dos ROV, com significância estatística (Tabela 2). Todavia, é importante destacar que, apesar de ter ocorrido significância estatística, os níveis de atividade elétrica apresentaram média dentro dos níveis considerados normais para o repouso, de acordo com a literatura, ou seja, em torno de $5 \mu \mathrm{V}^{16}$.

Quando comparados os músculos dos RN e $\mathrm{ROO}$, nas três situações avaliadas, verificou-se que os resultados se mostraram semelhantes entre os grupos. Exceto os músculos, orbicular inferior e temporal esquerdo, que foram significativamente mais ativos nos ROO durante o repouso e a isometria mastigatória, respectivamente.
A maior atividade elétrica evidenciada nos ROO, durante o repouso, no músculo orbicular inferior, indica que esse músculo se apresenta mais ativo, conforme mencionado anteriormente, e, por isso, necessita utilizar um maior número de fibras musculares para conseguir realizar o vedamento labial ${ }^{17}$; o que é observado através do aumento da atividade elétrica dessa musculatura, referenciado em outros estudos $^{10}$.

Quanto à maior atividade elétrica do músculo temporal esquerdo encontrada nos ROO, durante a isometria mastigatória, resultado semelhante foi verificado em estudo recente ${ }^{8}$, o qual explica que pelo fato de os respiradores orais apresentarem postura anteriorizada de cabeça, de modo a facilitar a passagem de ar pela orofaringe, os músculos temporais apresentam maior atividade elétrica na tentativa de compensar a menor atividade dos masseteres. Além disso, a maior atividade do músculo temporal esquerdo em relação ao direito pode estar relacionada ao padrão de preferência lateral mastigatória e à postura alterada de cabeça que comumente são verificados em respiradores orais ${ }^{8}$. Característica observada neste grupo durante avaliação clínica realizada.

Ao comparar a atividade elétrica dos músculos avaliados dos ROV com os ROO no repouso, na isometria mastigatória e na isometria labial, verificou-se que os resultados se mostraram semeIhantes entre os grupos, sem significância estatística. Isso indica que a etiologia da respiração oral não alterou o comportamento desses músculos no que diz respeito à atividade elétrica. Acredita-se que o padrão de atividade elétrica dos músculos 
avaliados nas situações de repouso, de isometria mastigatória e de isometria labial, possa estar mais relacionado à postura assumida pelos órgãos fonoarticulatórios para realizar a respiração oral do que à etiologia que a determina.

$\mathrm{Na}$ comparação dos músculos entre o mesmo lado e entre os lados da face, nos três grupos, durante as situações de repouso e de isometrias mastigatória e labial, verificou-se que, quando comparados os orbiculares superior e inferior, houve maior atividade do orbicular inferior durante $o$ repouso e a isometria labial, sendo que, no repouso, houve diferença altamente significativa nos ROV e nos ROO e, durante a isometria labial, houve diferença altamente significativa nos três grupos. Esses resultados mostram que os músculos orbiculares superior e inferior funcionam como entidades separadas e independentes ${ }^{17}$.

O resultado obtido para o repouso poderia ser justificado pelo fato do músculo orbicular inferior realizar maior esforço na tentativa de compensar a musculatura do orbicular superior que, nos respiradores orais, se encontra débili ${ }^{18}$. Para a isometria labial, esperava-se que apenas os dois grupos de respiradores orais apresentassem o músculo orbicular inferior mais ativo que o superior, o que poderia ser explicado pela dificuldade que eles apresentam para manter o vedamento labial ${ }^{10,15,16}$. No entanto, o músculo orbicular inferior apresentouse mais ativo inclusive nos RN. Esta compensação pode ter ocorrido pelo fato desta ser uma função que necessita maior força da região orbicular, e que possivelmente recruta a musculatura adjacente.

Ao comparar os músculos temporais direito e esquerdo e masseter direito e esquerdo, observouse uma assimetria entre os lados durante o repouso e a isometria mastigatória. No repouso, houve diferença significativa, com predominância de atividade elétrica do músculo temporal esquerdo no grupo de RN. Esse resultado concorda com estudo que apontou assimetria entre os lados em indivíduos normais, ao analisar o músculo masseter ${ }^{7}$. Tendo em vista que esses músculos atuam conjuntamente na função mastigatória, seria possível inferir que essa assimetria também ocorre no músculo temporal.

$\mathrm{Na}$ isometria mastigatória, verificou-se significância estatística em todos os grupos analisados, com predominância do músculo masseter direito; contudo, altamente significativa nos ROO. O resultado obtido para o grupo RN poderia ser justificado pela assimetria do músculo masseter, já verificada em indivíduos normais ${ }^{7}$. Outra possível justificativa seria o fato de as crianças deste grupo terem apresentado preferência mastigatória à direita, aspecto verificado na avaliação clínica do sistema estomatognático, já mencionada anteriormente; já que, o lado de preferência mastigatória tende a acarretar aumento na atividade elétrica da musculatura mastigatória no mesmo lado.

O resultado obtido para os grupos de respiradores orais vai ao encontro da literatura, que afirma ser evidente a assimetria na atividade elétrica muscular dos respiradores orais $7,8,19$. Essa assimetria pode ser explicada pela mastigação unilateral, que é freqüente nos respiradores orais ${ }^{20}$, e pela maior ocorrência de preferência mastigatória à direita, também verificada nos respiradores orais desta pesquisa, durante avaliação clínica realizada. No que se refere à maior significância estatística verificada nos ROO, é possível que as alterações estruturais decorrentes da obstrução respiratória ${ }^{18,19}$, tenham prejudicado seu desempenho na realização desta prova.

Na comparação entre os músculos masseter e temporal direito e masseter e temporal esquerdo na situação de repouso, foi verificado, no grupo de ROV, que os músculos temporais se apresentaram significativamente mais ativos que os masseteres. De modo semelhante, na comparação entre o masseter e o temporal, na isometria mastigatória, foi percebida significativa diferença nos três grupos avaliados neste estudo; todavia, percebe-se que foi altamente significativa no grupo de ROO (Tabela 3). Estes resultados também podem ser explicados pela postura anteriorizada de cabeça, frequentemente observada nos respiradores orais, alterando o equilíbrio da atividade elétrica entre estes músculos ${ }^{15}$. Além disso, outras hipóteses para justificar esse resultado seriam a tendência que estas crianças apresentam a um padrão de crescimento craniofacial vertical ${ }^{7}$ e o fato dos músculos temporais desempenharem função posicionadora da mandíbula, e portanto apresentarem maior atividade elétrica em relação aos masseteres no repouso ${ }^{19}$.

Poucos estudos realizam comparação entre indivíduos respiradores orais, caracterizando-os conforme a etiologia em obstrutivos e viciosos. A maioria dos estudos faz a comparação da atividade elétrica da musculatura facial entre respiradores nasais e respiradores orais, independente da etiologia, o que dificultou a discussão dos resultados.

Novos trabalhos com número maior de participantes e metodologia semelhante poderão fornecer subsídios para a comparação de informações e para conseqüentes benefícios a essas crianças, considerando as possíveis implicações clínicas das alterações musculares da respiração oral obstrutiva e viciosa. 


\section{CONCLUSÃO}

O modo respiratório não modificou o comportamento dos músculos faciais quando comparados os grupos RN, ROV e ROO, assim como, a etiologia da respiração oral não alterou a atividade elétrica dos músculos quando se comparou os grupo ROV e ROO. Os três grupos avaliados apresentaram assimetria da atividade elétrica muscular entre os lados da face e maior atividade elétrica do músculo temporal esquerdo, durante a isometria mastigatória; além de maior atividade do músculo orbicular inferior em relação ao superior durante a prova de isometria labial.

\begin{abstract}
Purpose: to check, through an electromyographic evaluation, the electrical activity pattern of the orbicular oral, masseter and temporal muscles on nasal breathers, obstructive and vicious oral breathers in order to compare them. Methods: 59 children, 15 nasal breathers (NB); 23 vicious oral breathers (VOB) and 21 obstructive oral breathers (OOB) were studied. All were submitted to an otorhinolaringological and speech-language patology evaluation and to an electromyographic exam in rest, masticatoric and labial isometries. We developed a Kruskal-Wallis variance analysis in order to make a comparison between the groups and the two-by-two groups and the Wilcoxon's test in order to compare between the muscles $(p<0,05)$. Results: after comparing the three groups, there were no relevant difference in the studied muscles, except for the right masseter muscle while resting, when the VOB and NB were compared; and to the lower orbicular muscle while resting and left temporal muscle in the masticatoric isometry, when NB and OOB were compared. In the comparison between $\mathrm{VOB}$ and $\mathrm{OOB}$, any muscle has showed a relevant difference. The lower orbicular muscle was more active than the upper orbicular muscle while resting and on labial isometry, mainly in VOB and OOB. The relation of the muscles on the opposed sides of face in the masticatoric isometry revealed asymmetry. The temporal muscle demonstrated being more active than the masseters while resting and in masticatoric isometry. Conclusion: the breathing mode did not modify the behavior of the evaluated muscles when comparing the groups; oral breathing etiology did not change the electrical activity of the evaluated muscles when comparing VOB and OOB.
\end{abstract}

KEYWORDS: Electromyography; Mouth Breathing; Facial Muscles

\section{REFERÊNCIAS}

1. Filho DI, Bertolini MM, Lopes ML. Contribuição multidisciplinar no diagnóstico e no tratamento das obstruções da nasofaringe e da respiração bucal. $R$ Clin Ortodon Dental Press. 2006; 4 (6): 90-102.

2. Rodrigues AMM, Bérzin F, Siqueira VCV. Análise eletromiográfica dos músculos masseter e temporal na correção da mordida cruzada posterior. Rev. Dental Press. Ortodon. Ortop. Facial. 2006; 11 (3): 55-62.

3. Cattoni DM, Fernandes FDM, Di Francesco RC, Latorre MRDO. Características do sistema estomatognático de crianças respiradoras orais: enfoque antroposcópico. Pró-Fono. 2007; 19(4): 347-51.

4. Hermens HJ, Freriks B, Disselhorst-Klug C, Rau G. Development of recommendations for SEMG sensors and sensor placement procedures. J. Electromyogr. and Kinesiol. 2000; 10(5): 361-74.
5. Barros JRC, Becker HMG, Pinto JA. Avaliação de atopia em crianças respiradoras bucais atendidas em centro de referência. J Pediatr. 2006; 82(6): 458-64.

6. Bianchini AP, Guedes ZCF, Hitos S. Respiração oral: causa x audição. Rev CEFAC. 2009; 11 (I1): 38-43.

7. Rahal A, Goffi-Gomez MVS. Estudo eletromiográfico do músculo masseter durante o apertamento dentário e mastigação habitual em adultos com oclusão dentária normal. Rev Soc Bras Fonoaudiol. 2009; 14(2):160-4.

8. Ferla A, Silva AT, Corrêa ECR. Atividade eletromiográfica dos músculos temporal anterior e masseter em crianças respiradoras bucais e em respiradoras nasais. Rev. Bras. Otorrinolaringol. 2008; 74 (4): 588- 95.

9. Biasotto DA. Estudo eletromiográfico dos músculos do sistema 
estomatognático durante a mastigação de diferentes materiais [dissertação mestrado]. Piracicaba: FOP/ UNICAMP; 2000.

10. Tosello DO, Vitti M, Bérzin F. EMG activity of the orbicularis and mentalis muscles in children with malocclusion, incompetent lips and atypical swallowing - Part II. Journal of oral rehabilitation. 1999; 26: 644-9.

11. De Luca, C.J. The use of surface electromyography in biomechamics. J Applied. Biom. 1997; 13(2): 135-63.

12. Ferrario VF, Sforza C, Jr Miani A, D'addona A, Barbini E. Electromyographic activity of human masticatory muscles in normal young people. Statistical evaluation of reference values for clinical applications. Journal of Oral Rehabilitation. 1993; 20:271-80.

13. Nagae, MH. Estudo eletromiográfico da correlação entre os músculos bucinador e masseter, durante a mastigação, em sujeitos Classe I e Classe III de Angle. Dissertação (Mestrado) - Faculdade de Odontologia de Piracicaba, Universidade Estadual de Campinas. Piracicaba, SP :[s.n.], 2005.

14. Frasson JMD, Maganani MBBA., Nouer F, Siqueira VCV, Lunardi N. Comparative
Cephalometric Study between nasal and predominantly mouth breathers. Rev. Bras Otorrinolaringol. 2006; 72(1): 72-81.

15. Lemos CM, Junqueira PAS, Goffi- Gomez MVS, Faria MEJ, Basso SC. Estudo da relação entre a oclusão dentária e a deglutição no respirador oral. Arq Int Otorrinolaringol. 2006; 10(2): 114-8.

16. Cram JR, Kasman GS, Holtz J. Introduction to Surface Electromyography. Maryland: Aspen Publishers, 1998.

17. Nieberg LG. An electromyographic and cephalometric radiographic investigation of the orofacial muscular complex. Am.J. Orthod. 1960; 46 (8): 627-8.

18. Vianna-Lara MS, Caria PHF. Electromyographic analysis of the upper lip in nose and mouth breathers. Braz J Oral Sci. 2006; 5(19): 120.

19. Oncins MC, Freire RMAC, Marchesan IQ. Mastigação: análise pela eletromiografa e eletrognatografia: seu uso na clínica fonoaudiológica. Distúrb Comun. 2006; 18(2):155-65.

20. Marchesan IQ. Motricidade oral: visão clínica integrada do trabalho fonoaudiológico integrado com outras especialidades. São Paulo: Pancast; 1993.
DOI: 10.1590/S1516-18462010005000136

RECEBIDO EM: 05/05/2010

ACEITO EM: 16/07/2010

Endereço para correspondência:

Luane de Moraes Boton

Rua Dr. Astrogildo de Azevedo, no 328 - Ap. 06,

Centro - Santa Maria - RS

CEP: 97015-150

E-mail: luaneboton@yahoo.com.br 